VOL. 167 , NO. 6 THE AMERICAN NATURALIST JUNE 2006

\title{
Sensory Drive in Cichlid Speciation
}

\author{
Martine E. Maan, ${ }^{1, \star}$ Kees D. Hofker, ${ }^{1, \dagger}$ Jacques J. M. van Alphen, ${ }^{1, \ddagger}$ and Ole Seehausen ${ }^{2, \$}$
}

1. Department of Animal Ecology, Institute of Biology, University of Leiden, P.O. Box 9516, 2300 RA Leiden, The Netherlands; 2. Department of Aquatic Ecology and Evolution, Institute of Zoology, University of Bern, Baltzerstrasse 6, CH-3012 Bern, Switzerland; and Eidgenössische Anstalt für Wasserversorgung, Abwasserreinigung, und Gewässerschutz (EAWAG) Ecology Research Centre, Seestrasse 79, CH-6047 Kastanienbaum, Switzerland

Submitted September 11, 2005; Accepted February 3, 2006; Electronically published April 13, 2006

AвSTRACT: The role of selection in speciation is a central yet poorly understood problem in evolutionary biology. The rapid radiations of extremely colorful cichlid fish in African lakes have fueled the hypothesis that sexual selection can drive species divergence without geographical isolation. Here we present experimental evidence for a mechanism by which sexual selection becomes divergent: in two sibling species from Lake Victoria, female mating preferences for red and blue male nuptial coloration coincide with their contextindependent sensitivities to red and blue light, which in turn correspond to a difference in ambient light in the natural habitat of the species. These results suggest that natural selection on visual performance, favoring different visual properties in different spectral environments, may lead to divergent sexual selection on male nuptial coloration. This interplay of ecological and sexual selection along a light gradient may provide a mechanism of rapid speciation through divergent sensory drive.

Keywords: cichlid fish, color evolution, sensory drive, sexual selection, speciation.

It is widely acknowledged that female mate choice for male ornamental traits can maintain and strengthen reproductive isolation between recently diverged species, even without postzygotic barriers to gene flow (Andersson 1994). The role of sexual selection in the emergence of repro-

\footnotetext{
* Corresponding author; e-mail: maan@rulsfb.leidenuniv.nl.

† E-mail: hofker@rulsfb.leidenuniv.nl.

₹ E-mail: alphen@rulsfb.leidenuniv.nl.

`E-mail: ole.seehausen@eawag.ch.
}

Am Nat 2006 Vol 167, pp 000-000 (c) 2006 by The University of Chicago 0003-0147/2006/16706-41296\$1500 All rights reserved ductively isolated populations, and hence in the speciation process itself, is less well understood and much more controversial (Arnegard and Kondrashov 2004). Especially when female choice selects for "good genes," it is hard to see how variation in mating preferences can evolve, let alone drive species divergence (Kirkpatrick and Nuismer 2004; but see Edelaar et al. 2004; Reinhold 2004). However, intraspecific variation in female preferences has been demonstrated in several taxa (e.g., Godin and Dugatkin 1995; Coleman et al. 2004), and more examples may emerge as research effort into the evolution of signal perception increases (Jennions and Petrie 1997).

The extremely species-rich cichlid communities of the East African lakes Malawi and Victoria have been presented as possible examples of speciation driven by sexual selection (Dominey 1984; McKaye 1991; Seehausen 2000; Danley and Kocher 2001). This hypothesis was inspired by the widespread occurrence of intra- and interspecific variation in male nuptial coloration and corresponding female mating preferences, often associated with only slight ecological differentiation and no intrinsic postzygotic isolation between species (Albertson et al. 1999; Seehausen 2000).

Haplochromine cichlids express considerable interspecific variation in color vision (van der Meer and Bowmaker 1995; Smit and Anker 1997). Seehausen et al. (1997) proposed that if such variation occurred within populations, together with directional sexual selection by female mate choice for brightly colored, conspicuous males, it might constitute disruptive sexual selection on male coloration and possibly cause speciation. Several recent studies have presented evidence for divergent sensory drive in species pairs that use visual signals in sexual communication (Boughman 2001; Fuller 2002; Leal and Fleishman 2004), indicating that it may be a potent driving force in speciation (Endler and McLellan 1988; Endler et al. 2001; Boughman 2002).

We investigate the divergence of a species pair that represents the dominant pattern of color variation among sympatric closely related Lake Victoria cichlids (Seehausen et al. 1999). Pundamilia pundamilia and Pundamilia nyererei are anatomically similar, but $P$. pundamilia males are metallic blue and P. nyererei males are bright red (fig. 1A). 

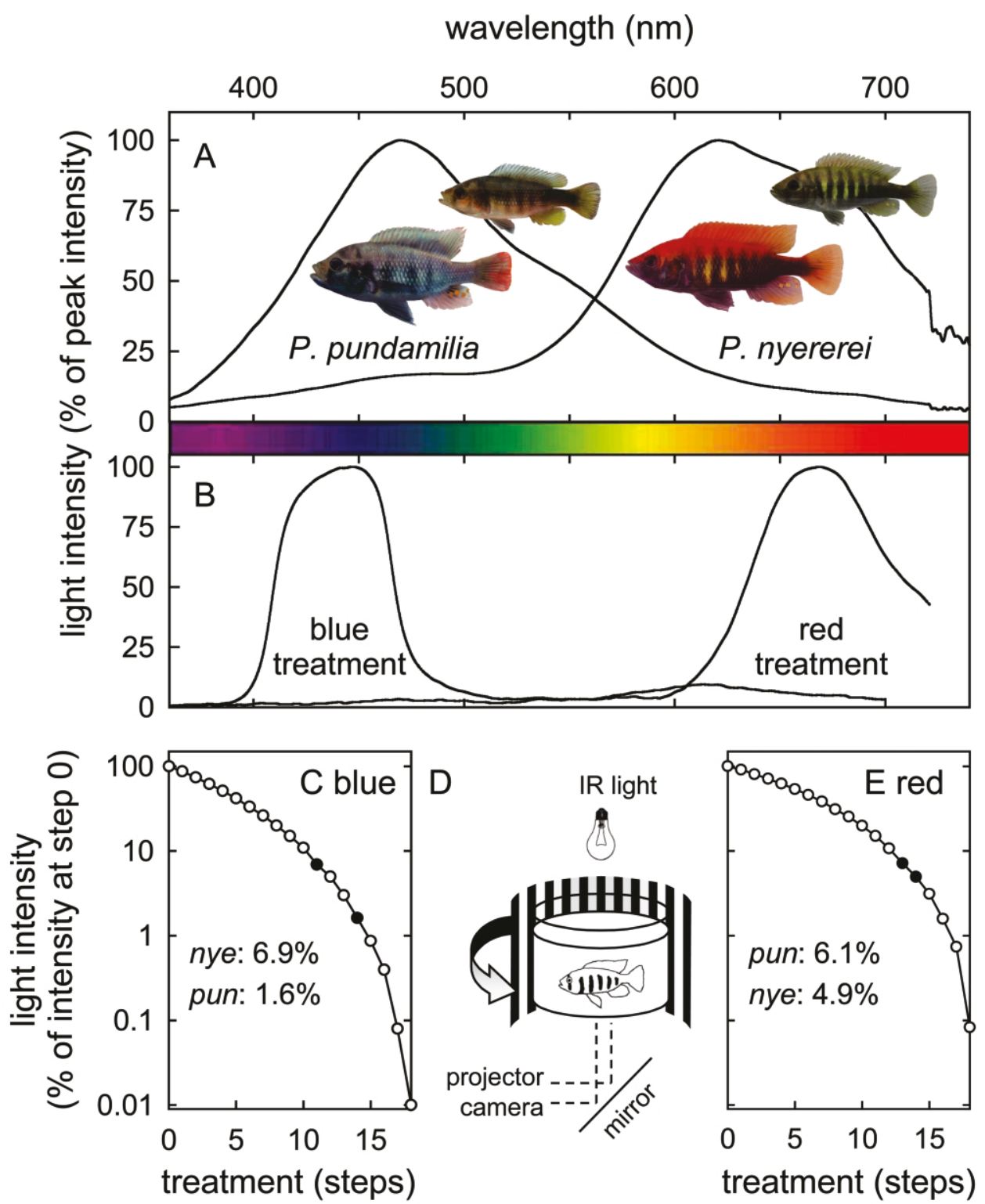

Figure 1: Male nuptial coloration in Pundamilia and the light treatments in the optomotor response test. A, Pundamilia pundamilia and Pundamilia nyererei males and females with the reflection spectra of male nuptial coloration. $B$, Transmission spectra of the blue and red light treatments used in the optomotor response test. $C, E$, Treatment light intensities, from step 0 to step 18 , expressed as a percentage of the treatment light intensity in step 0. Group medians for both species (filled symbols) are given in each panel. $D$, Experimental setup.

They behave as reproductively isolated species in some populations but interbreed in locations where the water is very turbid (Seehausen et al. 1997). Females of both species are cryptically colored and can be distinguished only with difficulty. They have assortative mating preferences and use male coloration as a choice criterion (Seehausen and van Alphen 1998). The hypothesis that sensory drive has been involved in the divergence of this species pair (Seehausen et al. 1997) predicts that the photic environments in the microhabitats of the two species differ, that the visual systems of the two species have diverged in adaptation to these different photic environments, and that, within a species, females prefer more conspicuous over less conspicuous males. The latter has been dem- 
onstrated in one of the two species: the red coloration of $P$. nyererei males is subject to directional sexual selection by female choice (Maan et al. 2004).

Here we report data consistent with divergent adaptation of the visual systems of the two species. First, we quantify the photic habitats of both species in nature. Although geographically $P$. nyererei is entirely sympatric with P. pundamilia, it breeds in deeper water (Seehausen and Bouton 1997). Because the turbid Lake Victoria waters selectively absorb and scatter light of short wavelengths, the intensity and spectrum of ambient light change rapidly with depth (De Beer 1989). Second, we test whether the two species differ in visual properties in the direction predicted by the difference in spectral environment. We use the optomotor response test to measure the contextindependent behavioral responses to colored light of both species.

\section{Methods}

The Fish

Fish were collected in February 2003 at Makobe Island (Seehausen and Bouton 1997) in the western Speke Gulf of Lake Victoria (Tanzania; water transparency: Secchi reading $=221 \pm 7 \mathrm{~cm}$ (mean $\pm \mathrm{SE}) ; 84$ measurements in 2000-2003). At this island, Pundamilia pundamilia and Pundamilia nyererei inhabit a continuous rocky slope from 0.5 to $7 \mathrm{~m}$ water depth. Pundamilia pundamilia is most abundant at $0.5-2 \mathrm{~m} ; P$. nyererei is most abundant at 4$7 \mathrm{~m}$. Both species occur at 2-4 $\mathrm{m}$ depth (Seehausen and Bouton 1997; M. E. Maan, personal observation). Their main habitats are not separated by any barrier (such as stretches of sand or mud), nor are they isolated by distance (the distances between locations within each habitat are not shorter than those between the habitats). Individuals of both species are regularly observed and caught in the entire $0.5-7-\mathrm{m}$ depth range.

Sensitivity thresholds were determined in OctoberDecember 2003. Because we were interested in correspondence between visual sensitivity and female preference, we used only females. Pundamilia nyererei females $(n=11)$ were caught in gill nets at a 4-7-m depth; $P$. pundamilia females $(n=14)$ were collected using hook and line at a $\sim 1-\mathrm{m}$ depth. Fish were shipped to the Netherlands and kept at $25^{\circ} \pm 2^{\circ} \mathrm{C}$ and a $12 \mathrm{~L}: 12 \mathrm{D}$ photoperiod. Tanks were illuminated with high-frequency fluorescent light tubes (Philips TLD $58 \mathrm{~W}$, light color 830, 1,100 lux). Shortly before an experiment, we measured fish standard length and inserted a microchip into the abdominal cavity for individual identification (12-mm glass tags, UKID122GL, Biomark); the species identity of the subjects was not known to the observer during the experiments.

\section{Spectrophotometry}

Reflection spectra of male nuptial coloration were measured using an Avantes PS 2048 spectrophotometer with SpectraWin 6.1 software (Avantes). We used one sexually active male of each species and measured reflectance in daylight in clear water, holding the probe of the spectrophotometer at a distance of $5 \mathrm{~mm}$ perpendicular to the fish skin and holding the fish underwater. Light reflectance was calibrated using a dark reference (covering the probe completely) and a light reference (reflectance off a white piece of plastic, also underwater); no additional illumination was used. For P. pundamilia, we measured reflectance off the blue sides of the fish; for $P$. nyererei, we measured the red dorsum (which is more important in female choice than the yellow coloration on the sides of the males; Maan et al. 2004). We normalized reflectance spectra by the peak reflectance to facilitate species comparison.

Treatment light spectra were measured using the same equipment and holding the probe in the middle of the experimental tank illuminated by the treatment light and filled with water to $15 \mathrm{~cm}$. With decreasing light intensity, the spectral curves became less steep, but the wavelength of maximum intensity did not change (wavelength of top $5 \%$ intensity [mean $\pm \mathrm{SE}$ ]: blue, $447.9 \pm 1.3 \mathrm{~nm}$; red, $670.2 \pm 0.7 \mathrm{~nm}$ ).

We measured light transmission at Makobe Island with an Ocean Optics PS 1000 spectrophotometer with a 10$\mathrm{m}$ optical fiber cable $(100 \mu \mathrm{m})$, using SpectraWin 4.16 software (Avantes). We quantified the spectral composition at different depths by calculating the "orange ratio," the light intensity in the 550-700-nm range (yellow, orange, red) divided by the intensity in the $400-550-\mathrm{nm}$ range (blue, green; Endler and Houde 1995).

\section{Optomotor Experiment}

The optomotor response test exploits the optokinetic response, the tendency of fish to follow a moving light stimulus. The method has been successfully employed in a variety of fish species (e.g., guppies: Anstis et al. 1998; zebrafish: Krauss and Neumeyer 2003; goldfish: Schaerer and Neumeyer 1996; sticklebacks: Boughman 2001; and cichlids: van der Meer 1994; Kröger et al. 2003). Fish were placed individually in a cylindrical tank (diameter $20 \mathrm{~cm}$, water depth $15 \mathrm{~cm}$; fig. $1 D$ ). A screen with vertical black and white bars (35 mm wide) rotated around the tank at $8.33 \mathrm{rpm}$. Trials started with an acclimatization period of 10 min, during which the bar pattern rotated and the tank 

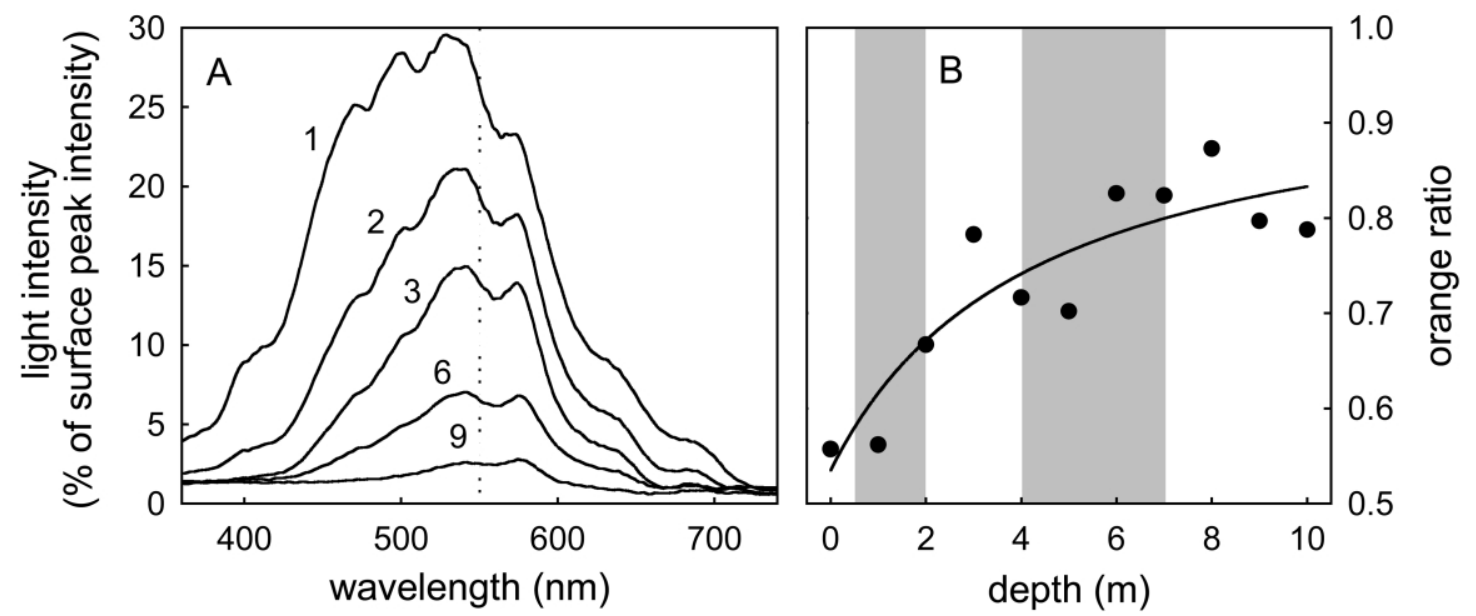

Figure 2: Photic habitats in the study population at Makobe Island. A, Transmission light spectra at different depths. Numbers indicate depth in meters. The dotted line indicates the 550-nm threshold that is used to calculate the orange ratio. $B$, Increase in orange ratio with water depth. Shaded areas indicate Pundamilia pundamilia $(0.5-2 \mathrm{~m})$ and Pundamilia nyererei $(4-7 \mathrm{~m})$ breeding habitats.

containing the test fish was illuminated with a small adapting light (230 lux), an infrared light source $(875 \mathrm{~nm}$, intensity $17 \mathrm{~mW} / \mathrm{sr}$; Agilent Technologies) above the tank, and the maximum intensity of treatment light. Treatment light (fig. $1 B$ ) was provided by a slide projector (GAF 131) with either a blue $(436 \mathrm{~nm})$ or a red $(656 \mathrm{~nm})$ narrowband filter (Spindler \& Hoyer). To narrow wavelength ranges, we added a colored Lee filter (113, magenta) and density filters (Lee 209; one density filter for the blue treatment and two for the red treatment). Light intensity was adjusted in 18 discrete steps by lowering the voltage feeding of the slide projector (fig. 1C, 1E). The response of the fish was observed using an infrared camera with a monitor. After the habituation period, the adapting light was switched off. If the fish continued following the rotating bar pattern within $15 \mathrm{~s}$ for at least $10 \mathrm{~s}$, the treatment light intensity was lowered by one step. This was repeated until the swimming response had disappeared. To confirm this threshold, the light intensity was increased by two steps and again lowered stepwise, this time reversing the rotating direction once at each light intensity level. The sensitivity threshold was defined as the lowest light intensity at which the fish responded to reversal of the rotation direction by reversing the swimming direction. The sensitivities that could be measured therefore ranged from 0 (maximum light intensity) to 18 (minimum light intensity). During the test procedure, the adapting light source was switched on for 1 min every $90 \mathrm{~s}$, to ensure that the fish were light adapted (Smit and Anker 1997). Each individual was tested five times in each light treatment, and the median sensitivity threshold of the test series was used for statistical analysis.

\section{Data Analysis}

Species differences were analyzed using independentsample $t$-tests (normal data) and Mann-Whitney $U$-tests (nonnormal data) in SPSS 10.0. To disentangle the effects of body size and species identity, we analyzed generalized linear models (GLMs) in R (http://www.r-project.org; Ihaka and Gentleman 1996) with a Poisson distribution. Also in $\mathrm{R}$, the orange ratio at Makobe Island was analyzed in GLMs with binomial distribution. Significance was determined by $F$-tests examining the change in deviance after removal of variables. Test statistics were adjusted for overdispersion and underdispersion (Venables and Ripley 2002).

\section{Results}

\section{The Photic Environment}

At Makobe Island, the proportion of long wavelengths (i.e., $>550 \mathrm{~nm})$ in the light spectrum increased rapidly with depth $(F=24.9, \mathrm{df}=1,9, P=.0008$; fig. 2$)$. As a result, the light spectrum in the habitat where Pundamilia nyererei dominates (4-7 $\mathrm{m}$ depth) was significantly more redshifted (orange ratio $=0.75$ ) than that in the habitat where Pundamilia pundamilia is most abundant $(0.5-2 \mathrm{~m}$ depth; orange ratio $=0.57): F=8.79, \mathrm{df}=1,4, P=.04$.

\section{The Sensitivities to Colored Light}

In both blue and red light, there were significant differences in optomotor response between the two species (fig. $3)$. In blue light, $P$. pundamilia females performed signif- 

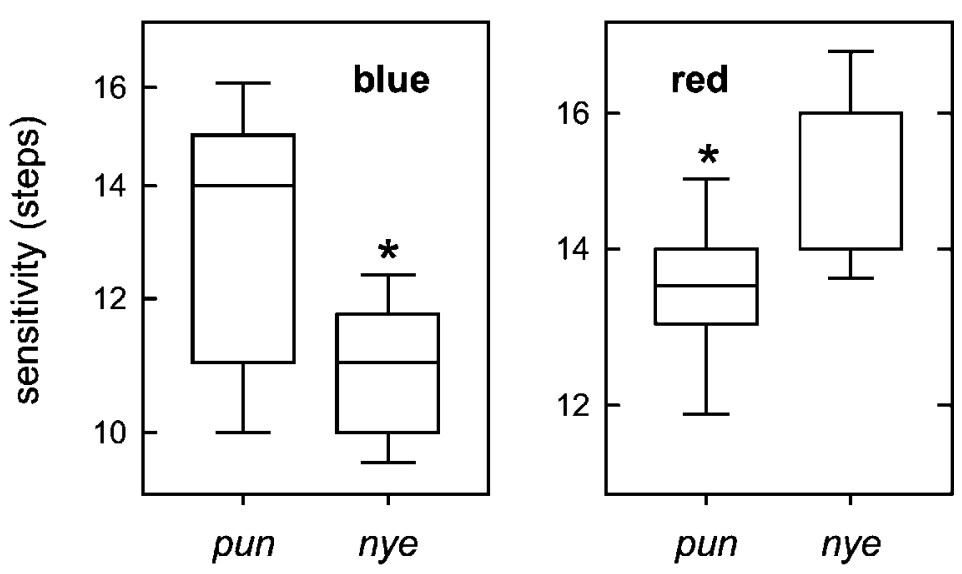

Figure 3: Performance of Pundamilia pundamilia (pun, $n=14$ ) and Pundamilia nyererei $(n y e, n=11)$ females in the optomotor response test. Left, Blue light; right, red light. Boxes represent medians with first and third quartiles; error bars indicate tenth and ninetieth percentiles; asterisks indicate $P<.02$.

icantly better than $P$. nyererei females $(n=14$ and 11 , respectively; Mann-Whitney $U$-test: $Z=2.86, P=.004$ ). In red light, the pattern was reversed $(Z=2.53, P=$ .011). The minimum intensity of blue light that $P$. pundamilia responded to was $2.7 \%$ of (or $1 / 37.7$ ) the minimum intensity of blue light that $P$. nyererei responded to. The minimum intensity of red light that $P$. nyererei responded to was $23.7 \%$ of (or $1 / 4.2$ ) the minimum intensity of red light that $P$. pundamilia responded to. Together, the blue and red optomotor response thresholds categorized 23 out of the 25 individuals tested into two nonoverlapping groups, one with individuals having high sensitivity to red light and low sensitivity to blue light (all P. nyererei) and the other with individuals having high sensitivity to blue light and low sensitivity to red light (all P. pundamilia). Thus, if mate choice in nature corresponds to visual sensitivities as measured by optomotor response thresholds, these results are consistent with assortative mating for 23 of 25 individuals (92\%).

On average, $P$. pundamilia females were larger than $P$. nyererei females (standard length $[\mathrm{mm}$, mean $\pm \mathrm{SE}]$ : P. pundamilia, $84.7 \pm 1.1$, and $P$. nyererei, $79.7 \pm 1.1$; independent-sample $t$-test: $t=3.11, \mathrm{df}=23, P=.005$; weight $(\mathrm{g})$ : $P$. pundamilia, $18.9 \pm 0.7$, and $P$. nyererei, $13.8 \pm 0.5 ; t=5.48, \mathrm{df}=23, P<.001)$. The performance in the experiment was not correlated with body size in either species (Spearman rank correlations between weight and standard length and red and blue sensitivity: all $r_{\mathrm{s}}<0.41$, $P>.21)$. GLMs including species, standard length, and weight showed that sensitivity threshold was best explained by species identity (red: estimate [species] $=0.11 \pm$ $0.036, F=9.47, \mathrm{df}=1,23, P=.0053$; blue: estimate [species] $=-0.23 \pm 0.061, F=14.11, \mathrm{df}=1,23, P=$
$.001)$. Models did not improve by including body size information (all $F<0.062$, df $=1,22, P>.81$ for the difference between models).

\section{Discussion}

We investigated the hypothesis that divergent natural selection on visual properties has contributed to the divergence of female mating preferences between sibling species of Lake Victoria cichlid fish, Pundamilia pundamilia and Pundamilia nyererei (Seehausen et al. 1997). This hypothesis was inspired by the observations that the species differ strikingly in male nuptial coloration, that the cryptically colored females use this difference as a basis for speciesassortative mate choice (Seehausen and van Alphen 1998), and that female mate choice exerts directional sexual selection on male coloration in at least one of the two species (P. nyererei; Maan et al. 2004). Moreover, the spectral characteristics of the Lake Victoria waters create a heterogeneous light environment (De Beer 1989) with potentially steep selection gradients.

We quantified the spectral habitats of $P$. pundamilia and P. nyererei at Makobe Island and found that the two species indeed inhabit significantly different light environments. Relative to the shallow-breeding P. pundamilia, $P$. nyererei experiences a narrower and redshifted ambient spectrum. In optomotor experiments, we tested whether this difference in light environment coincides with a difference in sensitivity to colored light. Our data demonstrate that the species indeed differ in behavioral responses to color stimuli outside the mate choice context: P. pundamilia females responded to lower intensities of blue light than did $P$. nyererei females. Conversely, P. nyererei females responded 
to lower intensities of red light than did P. pundamilia females.

Since we used wild-caught fish collected from different spectral environments, the differences we found could be due to phenotypic plasticity (Kröger et al. 2003). There are, however, strong indications of heritable differences in the visual systems of the two species. First, $P$. nyererei has a larger number of long-wavelength-sensitive (LWS) cones than P. pundamilia, and this difference is maintained in fish that are bred in identical laboratory conditions (Carleton et al. 2005). Second, the gene sequence of the LWS opsin gene differs between the species, corresponding to a 3-4-nm shift of maximum absorbance toward longer wavelengths in P. nyererei (Carleton et al. 2005).

Several earlier studies have demonstrated differences in absorbance spectra between cone pigments extracted from retinas of different haplochromine species (van der Meer and Bowmaker 1995), in cone opsin gene and protein sequences (Carleton and Kocher 2001; Terai et al. 2002), and in opsin gene expression (Carleton and Kocher 2001). Our study provides the first evidence that differences between sibling species in opsin protein sequences, opsin expression patterns (Carleton et al. 2005), and female mating preferences for different male colors (Seehausen and van Alphen 1998) are accompanied by differences in context-independent behavioral responses to light of different wavelengths. This is an important but previously untested prediction of the sensory-drive speciation hypothesis.

Many animals use only long-wavelength-sensitive (LWS) cones for the perception of movement (e.g., goldfish: Schaerer and Neumeyer 1996), and this may hold for cichlid fish as well (but see Kröger et al. 2003). In the case of Pundamilia, the difference in optomotor response between $P$. pundamilia and P. nyererei could be due to differences in expression level or in the peak sensitivity of a single cone pigment, most likely LWS (Carleton et al. 2005). By contrast, color assessment in the mate choice context may involve the joint action of all cone types (three different cone pigments). Further, behavioral responses to visual stimuli are mediated by information processing in the brain. For these reasons, further work is required to investigate how variation in Pundamilia optomotor responses relates to variation in female preferences for male nuptial coloration.

Either way, our data are consistent with the hypothesis that the visual systems of $P$. pundamilia and $P$. nyererei have evolved under divergent natural selection exerted by the spectral depth gradient in the natural habitat of the fish and that this in turn mediates divergent or disruptive sexual selection on male nuptial coloration. Increased sensitivity to light of longer wavelengths (longer wavelength of maximum sensitivity in the LWS cones, i.e., longer $\lambda_{\max }$, and/or a larger proportion of LWS-expressing cones) is advantageous in deeper water, while shorter $\lambda_{\max }$ and a larger proportion of short-wavelength-sensitive (SWS) cones are favored in shallow water. Consequently, if females prefer conspicuous males (Maan et al. 2004; Pauers et al. 2004), males that reflect mainly on the blue end of the spectrum have an advantage in shallow water, where selection favors shorter $\lambda_{\max }$ and a larger proportion of SWS-expressing cones. In deeper water, where selection favors longer $\lambda_{\max }$ and a larger proportion of LWSexpressing cones, red males are likely to have higher mating success.

Fish visual systems tend to be adapted to the spectral environment (Ali 1975; Loew and Lythgoe 1978; Levine and MacNichol 1982; van der Meer and Bowmaker 1995; Cummings and Partridge 2001; Jokela et al. 2003). The relationship between spectral environment and sexual color signals, however, is often negative (Marchetti 1993; Boughman 2001; Fuller 2002; Leal and Fleishman 2004): wavelengths that dominate in the ambient light spectrum are not used in visual signals. This maximizes color signal conspicuousness through background contrasting. In Pundamilia, however, male nuptial coloration exploits the available extremes in the ambient spectrum to maximize contrast against the green-yellow background light (fig. 2). Thus, the blue $P$. pundamilia inhabits a broad-spectrum environment where blue wavelengths are available to generate contrast. The red $P$. nyererei inhabits a redshifted photic environment and exploits the long-wavelength extreme of the ambient spectrum to maximize contrast against the background light. This difference with other taxa may be due to the steepness of the spectral gradient in Lake Victoria. In the P. nyererei habitat at Makobe Island, light intensity in the short-wavelength range is so low that blue signals are not conspicuous or are even invisible there.

We conclude that the interaction of divergent ecological selection on the visual system along a spectral light gradient and directional sexual selection for bright male color may result in divergent or disruptive sexual selection on male coloration through female mate choice. Consistent with sensory-drive speciation models (Endler 1992; Boughman 2002), this mechanism may have driven the divergence of $P$. pundamilia and $P$. nyererei. The geographically sympatric and ecologically parapatric distribution of the two species suggests that this process may have occurred without geographical isolation. The same mechanism is also likely to contribute to the maintenance of reproductive isolation after speciation. Given the abundance of blue-red sister species and polymorphisms among Lake Victoria cichlid fish (Seehausen et al. 1999), we postulate that similar mechanisms may have been involved in other cases of rapid speciation that resulted in sympatric 
sister species that differ in male nuptial coloration and depth distribution. Likewise, divergent selection on visual properties may have contributed to the cichlid radiations in other African lakes. In Lake Malawi, for example, which has a higher water transparency and shorter wavelength of maximum transmission than Lake Victoria, blue-yellow, rather than blue-red, species pairs or morphs predominate (McElroy et al. 1991; Deutsch 1997; Seehausen et al. 1999). This corresponds to patterns of opsin gene expression in these fish (Carleton and Kocher 2001).

\section{Acknowledgments}

We wish to thank the Tanzanian Fisheries Research Institute (P. O. J. Bwathondi, E. F. B. Katunzi) for hospitality and facilities, the Tanzanian Commission for Science and Technology for research permission, and M. Haluna, M. Kayeba, and H. D. J. Mrosso for field assistance. E. A. P. Ripmeester, H. J. van der Meer, and F. Witte provided valuable advice and practical help with the experimental design. We thank P. Hiddinga, H. D. J. Mrosso, and R. van der Kleij for their help with spectrophotometry, and K. L. Carleton, M. E. Cummings, R. C. Fuller, K. Kraaijeveld, T. D. Price, H. Slabbekoorn, T. J. M. Van Dooren, F. Witte, and one anonymous reviewer for comments and suggestions. The study was supported by the Netherlands Science Foundation (WOTRO 82-243) and the Dobberke Foundation.

\section{Literature Cited}

Albertson, R. C., J. A. Markert, P. D. Danley, and T. D. Kocher. 1999. Phylogeny of a rapidly evolving clade: the cichlid fishes of Lake Malawi, East Africa. Proceedings of the National Academy of Sciences of the USA 96:5107-5110.

Ali, M. A., ed. 1975. Vision in fishes: new approaches to research. Plenum, New York.

Andersson, M. B. 1994. Sexual selection. Princeton University Press, Princeton, NJ.

Anstis, S., P. Hutahajan, and P. Cavanagh. 1998. Optomotor test for wavelength sensitivity in guppyfish (Poecilia reticulata). Vision Research 38:45-53.

Arnegard, M. E., and A. S. Kondrashov. 2004. Sympatric speciation by sexual selection alone is unlikely. Evolution 58:222-237.

Boughman, J. W. 2001. Divergent sexual selection enhances reproductive isolation in sticklebacks. Nature 411:944-948.

- 2002. How sensory drive can promote speciation. Trends in Ecology \& Evolution 17:571-577.

Carleton, K. L., and T. D. Kocher. 2001. Cone opsin genes of African cichlid fishes: tuning spectral sensitivity by differential gene expression. Molecular Biology and Evolution 18:1540-1550.

Carleton, K. L., J. Parry, J. K. Bowmaker, D. M. Hunt, and O. Seehausen. 2005. Colour vision and speciation in Lake Victoria cichlid from the genus Pundamilia. Molecular Ecology 14:4341-4353.

Coleman, S. W., G. L. Patricelli, and G. Borgia. 2004. Variable female preferences drive complex male displays. Nature 428:742-745.

Cummings, M. E., and J. C. Partridge. 2001. Visual pigments and optical habitats of surfperch (Embiotocidae) in the California kelp forest. Journal of Comparative Physiology A 187:875-889.

Danley, P. D., and T. D. Kocher. 2001. Speciation in rapidly diverging systems: lessons from Lake Malawi. Molecular Ecology 10:10751086.

De Beer, M. 1989. Light measurements in Lake Victoria, Tanzania. Annales du Musée Royale de l'Afrique Centrale; Sciences Zoologiques (Tervuren, Belgium) 257:57-60.

Deutsch, J. C. 1997. Colour diversification in Malawi cichlids: evidence for adaptation, reinforcement or sexual selection? Biological Journal of the Linnean Society 62:1-14.

Dominey, W. J. 1984. Effects of sexual selection and life history on speciation: species flocks in African cichlids and Hawaiian Drosophila. Pages 231-249 in A. A. Echelle and I. Kornfield, eds. Evolution of fish species flocks. University of Maine Press, Orono. Edelaar, P., van Doorn, G. S., and Weissing, F. J. 2004. Sexual selection on good genes facilitates sympatric speciation. Pages 205-210 in G. S. van Doorn, Sexual selection and sympatric speciation. PhD thesis. University of Groningen.

Endler, J. A. 1992. Signals, signal conditions, and the direction of evolution. American Naturalist 139(suppl.):S125-S153.

Endler, J. A., and A. E. Houde. 1995. Geographic variation in female preferences for male traits in Poecilia reticulata. Evolution 49:456468.

Endler, J. A., and T. McLellan. 1988. The processes of evolution: toward a newer synthesis. Annual Review of Ecology and Systematics 19:395-421.

Endler, J. A., A. Basolo, S. Glowacki, and J. Zerr. 2001. Variation in response to artificial selection for light sensitivity in guppies (Poecilia reticulata). American Naturalist 158:36-48.

Fuller, R. C. 2002. Lighting environment predicts the relative abundance of male colour morphs in bluefin killifish (Lucania goodei) populations. Proceedings of the Royal Society of London B 269: 1457-1465.

Godin, J. G. J., and L. A. Dugatkin. 1995. Variability and repeatability of female mating preference in the guppy. Animal Behaviour 49: 1427-1433.

Ihaka, R., and R. Gentleman. 1996. R: a language for data analysis and graphics. Journal of Computational and Graphical Statistics 5:299-314.

Jennions, M. D., and M. Petrie. 1997. Variation in mate choice and mating preferences: a review of causes and consequences. Biological Reviews of the Cambridge Philosophical Society 72:283-327.

Jokela, M., A. Vartio, L. Paulin, N. Fyhrquist-Vanni, and K. Donner. 2003. Polymorphism of the rod visual pigment between allopatric populations of the sand goby (Pomatoschistus minutus): a microspectrophotometric study. Journal of Experimental Biology 206: 2611-2617.

Kirkpatrick, M., and S. L. Nuismer. 2004. Sexual selection can constrain sympatric speciation. Proceedings of the Royal Society of London B 271:687-693.

Krauss, A., and C. Neumeyer. 2003. Wavelength dependence of the optomotor response in zebrafish (Danio rerio). Vision Research 43:1273-1282.

Kröger, R. H. H., B. Knoblauch, and H. J. Wagner. 2003. Rearing in different photic and spectral environments changes the optomotor response to chromatic stimuli in the cichlid fish Aequidens pulcher. Journal of Experimental Biology 206:1643-1648.

Leal, M., and L. J. Fleishman. 2004. Differences in visual signal design 
and detectability between allopatric populations of Anolis lizards. American Naturalist 163:26-39.

Levine, J. S., and E. F. MacNichol Jr. 1982. Color vision in fishes. Scientific American 246:108-117.

Loew, E. R., and J. N. Lythgoe. 1978. Ecology of cone pigments in teleost fishes. Vision Research 18:715-722.

Maan, M. E., O. Seehausen, L. Soderberg, L. Johnson, E. A. P. Ripmeester, H. D. J. Mrosso, M. I. Taylor, T. J. M. Van Dooren, and J. J. M. van Alphen. 2004. Intraspecific sexual selection on a speciation trait, male coloration, in the Lake Victoria cichlid Pundamilia nyererei. Proceedings of the Royal Society of London B 271:2445-2452.

Marchetti, K. 1993. Dark habitats and bright birds illustrate the role of the environment in species divergence. Nature 362:149-152.

McElroy, D. M., I. Kornfield, and J. Everett. 1991. Coloration in African cichlids: diversity and constraints on Lake Malawi endemics. Netherlands Journal of Zoology 41:250-268.

McKaye, K. R. 1991. Sexual selection and the evolution of the cichlid fishes of Lake Malawi, Africa. Pages 241-257 in M. Keenleyside, ed. Behavior, ecology, and evolution of cichlid fishes. Chapman \& Hall, London.

Pauers, M. J., J. S. McKinnon, and T. J. Ehlinger. 2004. Directional sexual selection on chroma and within-pattern colour contrast in Labeotropheus fuelleborni. Proceedings of the Royal Society of London B 271:S444-S447.

Reinhold, K. 2004. Modeling a version of the good-genes hypothesis: female choice of locally adapted males. Organisms, Diversity, and Evolution 4:157-163.

Schaerer, S., and C. Neumeyer. 1996. Motion detection in goldfish investigated with the optomotor response is "color blind." Vision Research 36:4025-4034.

Seehausen, O. 2000. Explosive speciation rates and unusual species richness in haplochromine cichlid fishes: effects of sexual selection. Advances in Ecological Research 31:237-274.
Seehausen, O., and N. Bouton. 1997. Microdistribution and fluctuations in niche overlap in a rocky shore cichlid community in Lake Victoria. Ecology of Freshwater Fish 6:161-173.

Seehausen, O., and J. J. M. van Alphen. 1998. The effect of male coloration on female mate choice in closely related Lake Victoria cichlids (Haplochromis nyererei complex). Behavioral Ecology and Sociobiology 42:1-8.

Seehausen, O., J. J. M. van Alphen, and F. Witte. 1997. Cichlid fish diversity threatened by eutrophication that curbs sexual selection. Science 277:1808-1811.

1999. Can ancient colour polymorphisms explain why some cichlid lineages speciate rapidly under disruptive sexual selection? Belgian Journal of Zoology 129:43-60.

Smit, S. A., and G. C. Anker. 1997. Photopic sensitivity to red and blue light related to retinal differences in two zooplanktivorous haplochromine species (Teleostei, Cichlidae). Netherlands Journal of Zoology 47:9-20.

Terai, Y., W. E. Mayer, J. Klein, H. Tichy, and N. Okada. 2002. The effect of selection on a long wavelength-sensitive ( $L W S$ ) opsin gene of Lake Victoria cichlid fishes. Proceedings of the National Academy of Sciences of the USA 99:15501-15506.

van der Meer, H. J. 1994. Ontogenic change of visual thresholds in the cichlid fish Haplochromis sauvagei. Brain, Behavior and Evolution 44:40-49.

van der Meer, H. J., and J. K. Bowmaker. 1995. Interspecific variation of photoreceptors in four co-existing haplochromine cichlid fishes. Brain, Behavior and Evolution 45:232-240.

Venables, W. N., and B. D. Ripley. 2002. Modern applied statistics with S. Springer, New York.
Associate Editor: Michael J. Ryan Editor: Jonathan B. Losos 